\title{
Numerical Hydrodynamics: SPH versus AMR
}

\author{
Tomek Plewa \\ Nicolaus Copernicus Astronomical Center, Bartycka 18, 00-716 \\ Warsaw, Poland
}

\begin{abstract}
The advantages and disadvantages of two approaches to astrophysical hydrodynamics, Smoothed Particle Hydrodynamics (SPH) and Adaptive Mesh Refinement (AMR), are briefly discussed together with some current problems of computational hydrodynamics.
\end{abstract}

\section{From Henyey to SPH and AMR}

A history of numerical hydrodynamics in application to the problem of protostellar collapse begins more than three decades ago. It is remarkable to note that already at that time numerical simulations followed two distinct paths. Bodenheimer \& Sweigart (1968) and Bodenheimer (1968) used a Lagrangian implicit method closely following Henyey's approach (Henyey, Forbes \& Gould 1964) commonly used for stellar evolution computations. On the other hand, Larson (1969) developed a variant of an Eulerian scheme for radiation hydrodynamics in the optically thick regime. Despite the use of very different numerical schemes, both studies yielded very similar results.

However, problems like the massive removal of angular momentum from the clump of molecular gas collapsing into stellar densities, could not be addressed within the one-dimensional framework. Among the simplest ways of removing excess angular momentum is the deposition of angular momentum in the orbital motion of planets formed around a central star, or by the formation of a multiple stellar system. Neither of the two ways of removal of angular momentum can be studied in detail in one dimension. To overcome this problem Lucy (1977) proposed a method in which the distribution of hydrodynamical quantities is approximated with a discrete set of diffuse clouds of matter, Smoothed Particle Hydrodynamics (Monaghan 1992). The SPH method is purely Lagrangian, can be easily implemented on a computer, and allows for performing 3-dimensional simulations even on relatively small systems.

On the other hand, the solution of the hydrodynamical equations in multidimensions poses severe problems for grid based codes. In the Lagrangian approach the grid cells follow individual fluid elements and whenever a significant amount of shear is present in the flow the numerical grid becomes heavily distorted. This problem is nicely illustrated by the work of Woodward (1976) in his study of a shock-cloud interaction problem. Since a strong distortion of the computational grid leads to a loss of accuracy of the discretization, most of the later research on Lagrangian schemes was devoted to the development of so- 
phisticated rezoning modules which would allow to adequately follow individual fluid elements with an accuracy independent of their shapes.

An overall quality of the solution in the Eulerian model is determined by both the order of the advection scheme and the resolution provided by the numerical grid. Woodward and Colella (1984) demonstrated that substantial savings in terms of both memory and CPU time can be achieved when advection schemes of high-order are used instead of their low-order counterparts. These modern advection algorithms, shock-capturing schemes, gained much popularity during the last decade and follow the seminal works of Godunov (1959), van Leer (1976) and Harten (1983). The shock-capturing methods are characterized by at least a second order accuracy in the smooth part of the flow, with flow discontinuities being resolved in one or two zones, and no artificial viscosity is required to obtain a physically correct solution.

It took almost 20 years for Woodward's pioneering study of a shock-cloud interaction to be successfully addressed with an Eulerian code (Klein, McKee $\&$ Colella 1994). However, even the use of a shock-capturing scheme was still not enough to adequately resolve the flow structure and to obtain a convergent solution: a novel technique of local adaptive mesh refinement (AMR; Berger \& Colella 1989) was employed. In essence, AMR is a way of efficient discretization which allows to concentrate the computational effort in regions in which the errors of the solution are large. From a conceptual point of view AMR is similar to multigrid techniques with local refinements (Brandt 1984), in which the computational volume is covered with a hierarchy of completely nested grid patches and the resolution increases between parent and child grid levels by some predefined integer factor. However, due to the conservative character of the time-dependent hydrodynamical equations, the AMR method has to fulfill additional requirements making its actual computer implementation, to say the least, difficult.

\section{New Computers Required}

The planning of numerical simulations is much similar to the concept of project design known from industry rather than science. However, in both cases, given that objectives of the project have been clearly defined, a proper choice of the method of solution is necessary. Once an adequate tool is available one needs to find a way for using it. In practice this demand translates into having access to a computer installation which has to provide enough resources for obtaining the solution to the problem at hand. At first this last issue may appear to be a far side of numerical modelling, but to those involved in computationally intensive calculations it is this side which ultimately makes it possible to accomplish numerical simulation.

Large simulations represent a separate class of computational problems which are characterized by a high value of the product of computer memory and processor time. This practical definition follows the observation that simulations done on grids with just a few thousand zones (or particles) might be demanding once the modelled system has to be evolved for a long time. A typical example here are simulations of accretion disks which suffer from short evolutionary timescales present near the inner edge of the disk. On the other hand, 
large memory is required for virtually all three-dimensional problems, even with memory savings indirectly offered by modern advection methods, or whenever very high, even if only locally, resolution is needed. The case of planet formation in protoplanetary disks serves here as an example.

Furthermore, the large size of the simulation demands a certain minimum speed of the processor and the main memory: program data has to be accessed and subsequently processed within acceptable time. Most of the large simulations put much more strain on the memory subsystem, including the system bus, than on the processor and, therefore, are "bandwidth" rather than CPU limited. Certainly, not only some of those available but even the largest existing computer installation may not satisfy the requirement imposed by a "memory times processing speed" limit: numerical experiments are limited by the availability of computer resources. Finally, also storage and analysis of large $\left(\sim 10^{2}\right.$ times problem memory) data sets requires specialized hardware and software.

The variety of existing computer architectures does not necessarily make numerical simulations easier to plan and conduct. The performance of a computer code may differ vastly between scalar and vector architectures. Distributed and shared memory machines follow different programming paradigms, may ask for deep modifications to the existing codes. Such "technicalities" should be considered at the very beginning of any project which involves intensive computations as they might be difficult or impossible to overcome later. Keeping an eye wide open on current trends in computer architecture design, usually reflected in the policy of supercomputer centers, seems to be nowadays as important for numerical modelers as the development and application of new algorithms.

\section{A Few Critical Comments}

In my introductory remarks I sketched a path that astrophysical hydrodynamics followed from relatively simple one-dimensional Lagrangian models to full three-dimensional simulations done with help of the SPH and AMR methods. I find this development remarkable even though, as we could learn during this conference, after three decades the problem of one-dimensional stellar collapse may still offer enough of material for debates. As for the comparison between SPH and AMR, the two so extremely distinct approaches to hydrodynamics, there appears to exist a sharp boundary dividing the groups of users of these two methods. However, both methods have their own limits and aspects which ask for special attention. I will list them briefly.

The Lagrangian nature of the SPH method makes spatial resolution impossible to maintain comparable in all regions of the computational volume, a worry for those who aim at studying properties of voids in cosmological simulations. Resolution near the flow discontinuities is usually poor, equivalent to a few smoothing lengths ( particle "radii"), in most implementations of the SPH method shocks are handled with help of an artificial viscosity. Also the resolution and stability (in multidimensions) of contact discontinuities is problematic. SPH particles may "penetrate" each other increasing the diffusivity of the scheme and making studies which involve mixing and shear flows difficult.

Contrary to SPH, the resolution of an Eulerian model is defined by the smallest spatial scale and, would AMR not have been invented, no Eulerian 
calculation could achieve a resolution in mass comparable to that of large SPH simulations. However, the nonuniformity of the spatial resolution makes the AMR method unsuitable for studying turbulent flows which demand a smooth sampling of the spatial scales. Also, the roughness of the AMR grids adds a certain amount of vorticity to the model since each boundary between the levels in the AMR hierarchy appears as an obstacle to the flow and acts as a source of purely spurious vorticity.

Last but not least, the numerically "correct" results are those which correspond to the convergent solution. The Eulerian routine of doubling the resolution from one model to another and comparing low- and high-resolution models does not translate into doubling the number of particles in a SPH simulation. The concept of the smoothing length, usually mentioned when the spatial resolution of the SPH model is presented, is in this respect misleading since a twofold decrease of the smoothing length requires an increase in the number of neighbouring particles by a factor of $2^{D}$, where $D$ is the number of spatial dimensions. Yet, in most cases convergence studies (if present at all!) are done with the number of particles being varied by a factor of just a few.

I decided not to mention problems related to radiation transport or the incorporation of magnetic fields into hydrodynamical simulations. I did it on purpose since their complexity will likely add to the confusion; it is better to leave out their discussion for the years to come and closely follow the development of more efficient and correct algorithms. At this very moment it is my conviction that even pure hydrodynamics still poses challenging problems and obtaining physically meaningful results is, and as it has ever been, a difficult task.

Acknowledgments. I thank the organizing committee for financial support and hospitality during the conference. This work was partly supported by grant 2.P03D.014.19 from the Polish Committee for Scientific Research.

\section{References}

Berger, M. J., \& Colella, P. 1989, J. Comput. Phys., 82, 64

Bodenheimer, P. 1968, ApJ, 153, 483

Bodenheimer, P., \& Sweigart, A. 1968, ApJ, 152, 515

Brandt, A. 1984, Multigrid Techniques: 1984 Guide, with Applications to Fluid Dynamics, GMD-Studien Nr. 85, Sankt Augustin, GMD

Godunov, S. K. 1959, Mat. Sb., 47, 217

Harten, A. 1983, J. Comput. Phys., 49, 357

Henyey, L. G., Forbes, J. E., \& Gould, N. L. 1964, ApJ, 139, 306

Klein, R. I., McKee, C. F., \& Colella, P. 1994, ApJ, 420, 213

Larson, R. B. 1969, MNRAS, 145, 271

Lucy, L. B. 1977, AJ, 82, 1013

Monaghan, J. J. 1992, ARA\&A, 30, 543

van Leer, B. 1977, J. Comput. Phys., 23, 276

Woodward, P. R. 1976, ApJ, 207, 484

Woodward, P. R., Colella, P. 1984, J. Comput. Phys., 54, 115 